\title{
Suboptimal choice behavior by pigeons
}

\author{
Jessica P. Stagner and Thomas R. Zentall \\ University of Kentucky, Lexington, Kentucky
}

\begin{abstract}
Contrary to the law of effect and optimal foraging theory, pigeons show suboptimal choice behavior by choosing an alternative that provides $20 \%$ reinforcement over another that provides $50 \%$ reinforcement. They choose the $20 \%$ reinforcement alternative - in which $20 \%$ of the time, that choice results in a stimulus that always predicts reinforcement, and $80 \%$ of the time, it results in another stimulus that predicts its absence - rather than the $50 \%$ reinforcement alternative, which results in one of two stimuli, each of which predicts reinforcement $50 \%$ of the time. This choice behavior may be related to suboptimal human monetary gambling behavior, because in both cases, the organism overemphasizes the infrequent occurrence of the winning event and underemphasizes the more frequent occurrence of the losing event.
\end{abstract}

\begin{abstract}
Observing behavior can be defined as behavior that produces stimuli that signal whether reinforcement is available without directly affecting the overall probability of reinforcement. For example, Wyckoff (1952) reported that pigeons would step on a treadle, the sole function of which was to produce a discriminative stimulus that indicated what schedule was currently in effect on a response key. In other words, observing responses changed the schedule of reinforcement from a mixed to a multiple schedule. Similarly, pigeons prefer an alternative that produces discriminative stimuli (e.g., a red stimulus associated with $100 \%$ reinforcement on some trials or a green stimulus associated with extinction on other trials) over an alternative associated with an equal probability of reinforcement that produces stimuli (e.g., yellow on some trials, blue on others), both of which are associated with 50\% reinforcement (Roper \& Zentall, 1999; see also Dinsmoor, 1983).
\end{abstract}

One could conclude that the pigeons were showing a preference for the information provided by the discriminative stimuli. According to information theory, the amount of information transmitted is a function of the degree to which uncertainty is reduced. When, prior to the initial choice, the outcome is totally uncertain $(50 \%$ reinforcement) and, following the choice, the probability of reinforcement either increases to $100 \%$ or decreases to $0 \%$, uncertainty reduction should be maximal. Any increase or decrease in the overall probability of reinforcement should reduce the amount of information transmitted, because it would result in a decrease in uncertainty reduction. Consistent with information theory, Roper and Zentall (1999) showed that increasing the overall probability of reinforcement associated with the two alternatives decreases the preference for the alternative associated with the discriminative stimuli. On the other hand, contrary to information theory, they found that lowering the overall probability of reinforcement associated with both of the alternatives, which should also decrease the preference for the alternative that is followed by the discriminative stimuli, actually increases it.

According to the law of least effort, given equal probabilities of reinforcement, organisms should prefer alternatives that require less effort over those that require more effort. However, Roper and Zentall (1999) found that under conditions of equal reinforcement, pigeons were willing to expend considerably greater effort to obtain the discriminative stimuli (up to 16 pecks) than the nondiscriminative stimuli, for which only 1 peck was required. Given the strong preference for discriminative stimuli found by Roper and Zentall, we were prompted to ask whether pigeons would prefer an alternative that provides discriminative stimuli, even if there was a substantial cost involved in the form of loss of reinforcement.

According to the law of effect, given appropriate experience, organisms are presumed to favor alternatives that provide higher probabilities of reinforcement. Optimal foraging theory makes a similar prediction (Stephens $\&$ Krebs, 1986). But the preference for discriminative stimuli over nondiscriminative stimuli is not inconsistent with the law of effect, because the probability of reinforcement associated with the two alternatives is equal, and thus, it incurs no added cost to the animal in loss of reinforcement.

The results of early research involving differential probability of reinforcement have not been conclusive. When pigeons were given a choice between an alternative that provided $50 \%$ reinforcement with discriminative stimuli (sometimes, one color associated with $100 \%$ reinforcement and, other times, a different color associated with $0 \%$ reinforcement) and an alternative that provided a stimulus associated with $100 \%$ reinforcement, pigeons' choice was quite variable (some pigeons prefer one alternative,

T. R. Zentall, zentall@uky.edu 
whereas others prefer the other; Belke \& Spetch, 1994; Fantino, Dunn, \& Meck, 1979; Mazur, 1996; Spetch, Belke, Barnet, Dunn, \& Pierce, 1990; Spetch, Mondloch, Belke, \& Dunn, 1994).

In a more recent study, when choice of one alternative provided $75 \%$ reinforcement and choice of the other provided only $50 \%$ reinforcement but was followed by discriminative stimuli (one followed by $100 \%$ reinforcement, the other by $0 \%$ reinforcement), pigeons showed a reliable (69\%) preference for the $50 \%$ reinforcement alternative (Gipson, Alessandri, Miller, \& Zentall, 2009).

In the previous experiments, the probability of reinforcement for either alternative was generally quite high (at least 50\%). Paradoxically, however, Roper and Zentall (1999) reported that when the probability of reinforcement associated with the two alternatives was equal, the degree of preference for the alternative that resulted in discriminative stimuli varied inversely with the overall probability of reinforcement. That is, when the overall probability of reinforcement was low $(12.5 \%)$, preference for the discriminative stimulus alternative was very high $(98 \%)$, whereas when the overall probability of reinforcement was high $(87.5 \%)$, preference for the discriminative stimulus alternative was much more modest (68\%). Thus, in the present research, we asked whether pigeons would show an even stronger preference for an alternative associated with an even lower probability of reinforcement if that alternative provided discriminative stimuli signaling reinforcement and nonreinforcement. Specifically, we gave pigeons a choice between an alternative associated with a low probability of reinforcement $(20 \%)$ that provided discriminative stimuli (a stimulus associated with $100 \%$ reinforcement that occurred on $20 \%$ of the trials or a stimulus associated with $0 \%$ reinforcement that occurred on $80 \%$ of the trials) and an alternative associated with a higher probability of reinforcement $(50 \%)$ that did not provide discriminative stimuli (both stimuli were associated with $50 \%$ reinforcement). That is, would pigeons prefer an alternative associated with a low rate of reinforcement over an alternative associated with reinforcement 2.5 times as often?

\section{METHOD}

\section{Subjects}

The subjects were 8 White Carneaux pigeons that were retired breeders ( $8-10$ years old). Throughout the experiment, the pigeons were maintained at $85 \%$ of their free-feeding weight. They were individually housed in wire cages, with free access to water and grit, in a colony room that was maintained on a 12:12-h light:dark cycle.

\section{Apparatus}

The experiment was conducted in a BRS/LVE (Laurel, MD) sound-attenuating standard operant test chamber (see Gipson et al., 2009 , for details).

\section{Procedure}

Pretraining. Each pigeon was trained to peck each of five colors (red, yellow, green, blue, and white) for reinforcement on the left and right response keys and to peck a vertical line on the center key. Each pigeon then was trained to peck each of four colors (red, green, blue, and yellow) on the side keys on a fixed-interval 10-sec schedule (the first response after $10 \mathrm{sec}$ was reinforced).

Training. In training, all trials began with the vertical-line stimulus presented on the center key. On forced trials, a single peck to the vertical stimulus illuminated a white key on either the left or right side. The other side key remained dark. One peck to the illuminated key initiated a 10-sec colored stimulus of fixed duration. If the white key indicated that the discriminative-stimulus alternative was in effect, on $20 \%$ of the trials, a peck replaced the white key with one stimulus (A), and after $10 \mathrm{sec}$, reinforcement was provided. On the remaining $80 \%$ of the trials with that alternative, a peck replaced the white key with a different stimulus (B), and after $10 \mathrm{sec}$, the trial ended without reinforcement. Thus, for that alternative, reinforcement occurred $20 \%$ of the time.

If on forced trials the white key indicated that the alternative with nondiscriminative stimuli was in effect, on $20 \%$ of the trials a peck replaced the white key with a third stimulus (C), and after $10 \mathrm{sec}$, reinforcement was provided $50 \%$ of the time. On the remaining $80 \%$ of the trials with that alternative, a peck replaced the white key with the fourth stimulus (D), and after $10 \mathrm{sec}$, reinforcement was provided $50 \%$ of the time as well. Thus, for the second alternative, reinforcement occurred $50 \%$ of the time, whichever stimulus was presented.

The side keys on which the two alternatives appeared were counterbalanced, as were the colors associated with Stimuli A, B, C, and $\mathrm{D}$. There were 40 randomly alternating forced trials in each session (20 to the left and 20 to the right), with 4 Stimulus A trials, 16 Stimulus B trials, 4 Stimulus C trials, and 16 Stimulus D trials. A schematic of the design of the experiment is presented in Figure 1A.

For all pigeons, randomly mixed among the 40 forced trials were 20 choice trials per session. These trials also began with the vertical line on the center key. A peck to the vertical line illuminated both the left and right keys, and a single peck to either side key turned on (for $10 \mathrm{sec}$ ) one of the two colors associated with that alternative in the same proportion and with the same outcome as on forced trials. The unchosen white side key was darkened The intertrial interval was $10 \mathrm{sec}$. All of the pigeons received 12 sessions of training.

Reversal training. To rule out preferences on the basis of preexisting side biases, rather than reinforcement contingencies, the stimuli and reinforcement contingencies associated with the two sides were reversed for 12 sessions.

Shape discrimination training. To further test the pigeons' preference for the contingencies and to determine whether the pigeons would follow a discriminative stimulus at the time of choice, we replaced the white stimuli with two shapes, a circle and a plus sign, either of which could appear on the left or the right side on forced and choice trials from trial to trial. One shape was followed by the color associated with $100 \%$ reinforcement on some trials (on $20 \%$ of the trials) and with the color associated with $0 \%$ reinforcement on the remaining trials (on $80 \%$ of the trials), and the other shape was followed by one of the colors associated with $50 \%$ reinforcement overall. The shapes associated with the different probabilities of reinforcement were counterbalanced across pigeons.

Training with no discriminative color stimuli. Finally, to demonstrate that in the absence of discriminative stimuli predictive of $100 \%$ and $0 \%$ reinforcement, the pigeons would now choose the higher probability of reinforcement over the lower probability of reinforcement, the percentages of reinforcement associated with the colors were altered: The color associated with $100 \%$ reinforcement was decreased to $20 \%$, and the color associated with $0 \%$ reinforcement was raised to $20 \%$. The frequency of the colors remained the same; thus, the net result of the choice of initial shape stimuli remained $20 \%$ reinforcement for one alternative and $50 \%$ reinforcement for the other alternative (see Figure 1B). 
A

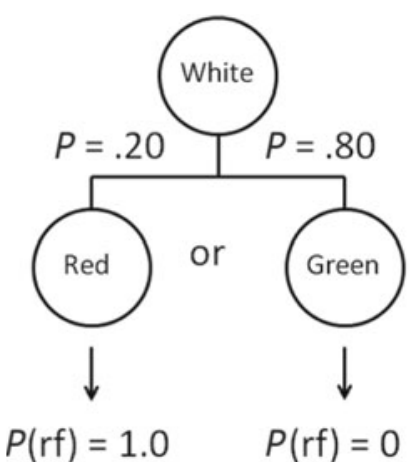

Training

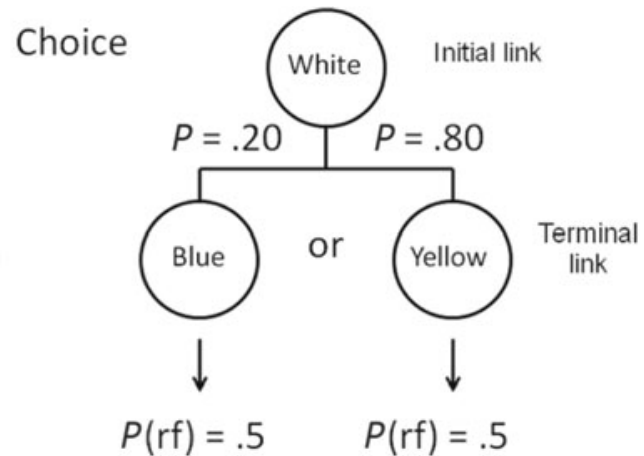

B No Differential Conditioned Reinforcement

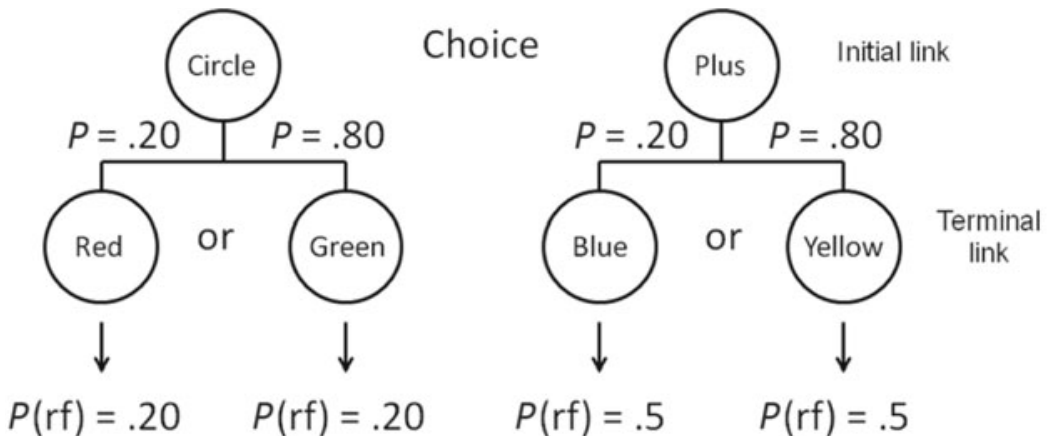

Figure 1. (A) Training: Choice trials in which one alternative, associated with $20 \%$ reinforcement, led to a signal for reinforcement on $20 \%$ of the trials and a signal for the absence of reinforcement on $80 \%$ of the trials (overall $20 \%$ reinforcement) and in which the other alternative led to either of two signals, one on $20 \%$ of the trials that led to a signal for $50 \%$ reinforcement, the other on $80 \%$ of the trials that led to a signal for $50 \%$ reinforcement (overall $50 \%$ reinforcement). The sides and colors were counterbalanced across pigeons. (B) No differential conditioned reinforcement: Choice trials in which choice of one alternative always led to a stimulus associated with $20 \%$ reinforcement and in which the other alternative always led to a stimulus associated with $50 \%$ reinforcement. The shapes and colors were counterbalanced across pigeons.

\section{RESULTS}

\section{Training}

The pigeons quickly acquired a preference for the alternative associated with $20 \%$ reinforcement (see Figure 2, Training). On the 12th session of training, the mean choice of the alternative associated with $20 \%$ reinforcement was $96.9 \%(S E M=1.0 \%)$ and significantly different from chance $[t(7)=18.83, p<.0001]$.

\section{Reversal Training}

During reversal training, the pigeons quickly reversed their preference (see Figure 2, Reversal). On the 12th session of training with the reversed configuration, the mean choice of the alternative associated with $20 \%$ reinforcement was $87.5 \%(S E M=6.9 \%)$ - a level that was significantly different from chance $[t(7)=5.54, p=.0009]$.

\section{Shape Discrimination Training}

When shapes were introduced as discriminative stimuli associated with the two alternatives, the pigeons quickly acquired a preference for the shape associated with $20 \%$ reinforcement (see Figure 2, Discrimination). On the 16th session of shape discrimination training, the mean choice of the stimulus shape associated with $20 \%$ reinforcement was $88.1 \%(S E M=6.9 \%)$-a level that was significantly different from chance $[t(7)=5.49$, $p=.0009]$.

\section{Training With No Discriminative Color Stimuli}

When the probability of reinforcement associated with the two colors that were presented following choice of the $20 \%$ reinforcement alternative was equated at $20 \%$, the pigeons dramatically reversed their preference, and the mean choice of the stimulus associated with $20 \%$ reinforcement on Session 20 was only $12.2 \%$ (SEM $=5.4 \%$; see Figure 2, No Differential Conditioned Reinforcement) - a level that was significantly different from chance $[t(7)=12.57, p<.0001]$. Thus, when reinforcement was not differentially signaled, the pigeons preferred the alternative associated with a higher probability of reinforcement. 


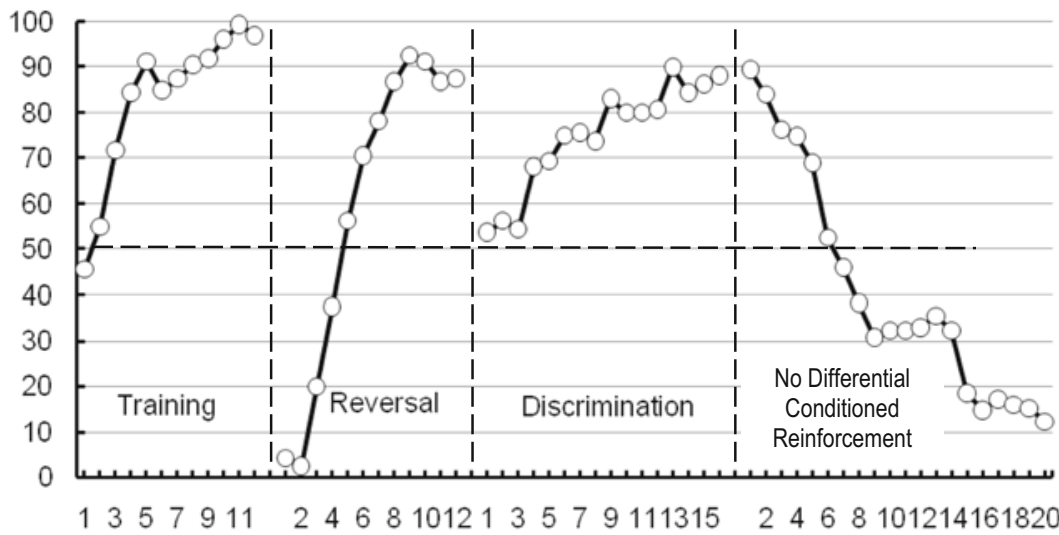

Sessions

Figure 2. Acquisition of the preference for an alternative that led to $20 \%$ reinforcement over an alternative that led to $\mathbf{5 0} \%$ reinforcement (Training), reversal of contingencies associated with each side (Reversal), training with a distinctive shape associated with the $20 \%$ and the 50\% reinforcement contingency (Discrimination) (see Figure 1B), and removal of the differential reinforcement associated with the stimuli associated with $20 \%$ reinforcement (No Differential Conditioned Reinforcement).

\section{DISCUSSION}

In the present experiment, pigeons showed a suboptimal preference for $20 \%$ reinforcement over $50 \%$ reinforcement (a choice that led to a loss of $60 \%$ of the food that could be obtained). This choice appears to depend on the very strong attraction of the signal for $100 \%$ reinforcement (a strong conditioned stimulus), which is particularly attractive, even though it occurs for those choices only $20 \%$ of the time. On the other hand, the signal for the absence of reinforcement does not appear to inhibit those choices (there is little conditioned inhibition), even though nonreinforcement occurs for those choices $80 \%$ of the time. The result is that the pigeons will sacrifice considerable opportunity for reinforcement to obtain discriminative stimuli if one of them predicts reinforcement with a high probability. When the $20 \%$ reinforcement alternative was shifted to the other side, the preference shifted to the other side as well, and when the $20 \%$ reinforcement was signaled by a shape, the pigeons followed the shape that predicted the lower probability of reinforcement. However, when the colors that followed choice of the $20 \%$ reinforcement alternative no longer differentially predicted reinforcement, in keeping with the law of effect, the pigeons preferred the alternative that led to $50 \%$ reinforcement over the alternative that led to $20 \%$ reinforcement.

One way to view the preference for a lower over a higher probability of reinforcement is in terms of the discrepancy between the expected probability of reinforcement at the time of the initial choice and the probability of reinforcement following that choice. In the present experiment, reinforcement was expected $20 \%$ of the time for the alternative that led to the discriminative stimuli. But appearance of the signal for the high payoff resulted in an increase in the expected percentage of reinforcement from $20 \%$ expected to $100 \%$ obtained (or an $80 \%$ increase from expectation), whereas presentation of the signal for the absence of reinforcement resulted in a decrease in the expected percentage of reinforcement from $20 \%$ to $0 \%$ (or only a $20 \%$ decrease in expectation). And even though the signal for the absence of reinforcement occurred four times as often as the signal for the high payoff, the large contrast associated with the high payoff appears to have had a much greater positive effect than the more frequent $0 \%$ payoff had a negative effect. On the other hand, for the alternative that did not provide discriminative stimuli, reinforcement was expected $50 \%$ of the time, but presentation of either of the stimuli that followed did not result in any change from expectation.

Consistent with this interpretation is the finding that in Pavlovian conditioning, when a conditioned stimulus is presented infrequently (i.e., a stimulus associated with a high probability of reinforcement is presented in a context associated with a low probability of reinforcement), it results in more rapid and more reliable conditioning than if the conditioned stimuli occur often (Jenkins, Barnes, \& Barrera, 1981).

As was mentioned earlier, pigeons show a small preference for an alternative associated with 50\% reinforcement with discriminative stimuli over an alternative associated with $75 \%$ reinforcement without discriminative stimuli (a loss of 33\% of the possible food obtainable). Paradoxically, in the present experiment, the pigeons showed a much larger preference for the alternative associated with a lower probability of reinforcement, in spite of the fact that it meant a loss of $60 \%$ of the possible food obtainable. Thus, it appears that it is the change 
in the probability of reinforcement relative to what is expected that is responsible for this effect.

The present results also fail to support an information theory account of the preference for the alternative associated with discriminative stimuli. According to information theory, reducing the probability of reinforcement from $50 \%$ (in Gipson et al., 2009) to 20\% in the present experiment should have reduced the information provided by the discriminative stimuli, because signals for reinforcement and nonreinforcement should be most informative when without them there would be maximum ambiguity (i.e., $50 \%$ reinforcement). Thus, reducing the overall probability of reinforcement should have reduced the preference for the alternative leading to the discriminative stimuli. Instead, the fact that the preference for the alternative associated with the discriminative stimuli actually increased offers added support for the conditioned reinforcement hypothesis.

Finally, the results of the present experiment may have implications for some forms of human gambling behavior-for example, the purchase of a lottery ticket. In both cases, the infrequent occurrence of a high-probability payoff may provide an added incentive that is not overcome by the much more frequent occurrence of a loss. Although other factors may play a role in human gambling behavior, relatively simple conditioning mechanisms - the asymmetrically large effect of an occasional conditioned reinforcer together with the relatively small effect of a frequent conditioned inhibitor-may be involved as well.

\section{AUTHOR NOTE}

This research was supported by National Institute of Mental Health Grant 63726 and by National Institute of Child Health and Development Grant 60996. We thank William Forward, Bradley Morris, Shannon Zentall, and Neal Singer for their comments on an earlier version of the manuscript. Correspondence should be addressed to T. R. Zentall, Department of Psychology, University of Kentucky, Lexington, KY 405060044 (e-mail: zentall@uky.edu).

\section{REFERENCES}

Belke, T. W., \& Spetch, M. L. (1994). Choice between reliable and unreliable reinforcement alternatives revisited: Preference for unreliable reinforcement. Journal of the Experimental Analysis of Behavior, 62, 353-366

DinSMOOR, J. A. (1983). Observing and conditioned reinforcement. Behavioral \& Brain Sciences, 6, 693-728.

Fantino, E., Dunn, R., \& Meck, W. (1979). Percentage reinforcement and choice. Journal of the Experimental Analysis of Behavior, 32, 335-340.

Gipson, C. D., Alessandri, J. J. D., Miller, H. C., \& Zentall, T. R. (2009). Preference for $50 \%$ reinforcement over $75 \%$ reinforcement by pigeons. Learning \& Behavior, 37, 289-298.

Jenkins, H. M., Barnes, R. A., \& Barrera, F. J. (1981). Why autoshaping depends on trial spacing. In C. M. Locurto, H. S. Terrace, \& J. Gibbon (Eds.), Autoshaping and conditioning theory (pp. 255-284). New York: Academic Press.

Mazur, J. E. (1996). Choice with certain and uncertain reinforcers in an adjusting delay procedure. Journal of the Experimental Analysis of Behavior, 66, 63-73

RoPer, K. L., \& ZenTALL, T. R. (1999). Observing behavior in pigeons: The effect of reinforcement probability and response cost using a symmetrical choice procedure. Learning \& Motivation, 30, 201-220.

Spetch, M. L., Belke, T. W., Barnet, R. C., Dunn, R., \& Pierce, W. D. (1990). Suboptimal choice in a percentage-reinforcement procedure: Effects of signal condition and terminal link length. Journal of the Experimental Analysis of Behavior, 53, 219-234.

Spetch, M. L., Mondloch, M. V., Belke, T. W., \& Dunn, R. (1994). Determinants of pigeons' choice between certain and probabilistic outcomes. Animal Learning \& Behavior, 22, 239-251.

Stephens, D. W., \& Krebs, J. R. (1986). Foraging theory. Princeton, NJ: Princeton University Press.

WYCKOFF, L. B., JR. (1952). The role of observing responses in discrimination learning: Part I. Psychological Review, 59, 431-442.

(Manuscript received October 2, 2009; revision accepted for publication January 1, 2010.) 